\title{
Advances in neuroimaging to support translational medicine in dementia
}

\author{
Thomas Edmund Cope (D) , 1,2,3 Rimona Sharon Weil (D) , 4,5,6,7 Emrah Düzel, ${ }^{8,9,10,11}$ \\ Bradford C Dickerson, ${ }^{12,13}$ James Benedict Rowe (1) 1,2,3
}

- Additional material is published online only. To view please visit the journal online (http://dx.doi.org/10.1136/ jnnp-2019-322402).

For numbered affiliations see end of article.

\section{Correspondence to} Dr Thomas Edmund Cope, Department of Clinical Neurosciences, University of Cambridge, Cambridge CB2 OSZ,UK; thomascope@gmail. com

Received 8 September 2020 Revised 17 November 2020 Accepted 18 November 2020 Published Online First 10 February 2021

\begin{abstract}
Advances in neuroimaging are ideally placed to facilitate the translation from progress made in cellular genetics and molecular biology of neurodegeneration into improved diagnosis, prevention and treatment of dementia. New positron emission tomography (PET) ligands allow one to quantify neuropathology, inflammation and metabolism in vivo safely and reliably, to examine mechanisms of human disease and support clinical trials. Developments in MRIbased imaging and neurophysiology provide complementary quantitative assays of brain function and connectivity, for the direct testing of hypotheses of human pathophysiology. Advances in MRI are also improving the quantitative imaging of vascular risk and comorbidities. In combination with large datasets, open data and artificial intelligence analysis methods, new informatics-based approaches are set to enable accurate single-subject inferences for diagnosis, prediction and treatment that have the potential to deliver precision medicine for dementia. Here, we show, through the use of critically appraised worked examples, how neuroimaging can bridge the gaps between molecular biology, neural circuits and the dynamics of the core systems that underpin complex behaviours. We look beyond traditional structural imaging used routinely in clinical care, to include ultrahigh field MRI (7T MRI), magnetoencephalography and PET with novel ligands. We illustrate their potential as safe, robust and sufficiently scalable to be viable for experimental medicine studies and clinical trials. They are especially informative when combined in multimodal studies, with model-based analyses to test precisely defined hypotheses.
\end{abstract}

\section{INTRODUCTION}

Brain imaging can bridge the gap between the progress made in understanding the cellular genetics and molecular biology of neurodegeneration ${ }^{1 \mathrm{w} 61-63}$ and clinical trials of novel interventions for dementia (box 1). The success of such translational medicine will be measured in terms of better diagnosis, treatment and ultimately prevention.

For diagnosis, improving current clinical practice requires quantitative methods that are accurate in terms of individual disease processes, and allow precision medicine by accommodating the complex multidimensionality of dementia. This multidimensionality is recognised in psychiatry in the Research Domain Criteria Criteria, ${ }^{2}$ providing a conceptual framework to integrate pathophysiology and symptomatology in spectral features of disease, rather than arbitrary categories. The spectral nature of dementia phenotypes is increasingly recognised in trans-diagnostic cohort studies ${ }^{3 \mathrm{w} 6465}$ and recent diagnostic criteria that encompass phenotypic variants. $^{4 w 66-71}$

For treatment trials, there are complementary roles of imaging. Imaging can identify individuals who are well but at-risk for dementia, for genetic or unknown reasons, tracking latent or premanifest pathology. ${ }^{5-7 w 72-74}$ Imaging also supports experimental medicine studies, with surrogate markers of disease processes as treatment outcomes. These secondary outcomes include evidence of drug target engagement, with diverse measures of neuropathology, neurophysiology, connectivity and function.

For prevention, imaging allows insight into modifiable disease processes including neurochemistry, physiology, molecular pathology and structure, and how these interact with the environment and genetics. While no single imaging modality quantifes the whole cascade of events from root cause to final phenotype, combinations of imaging methods can connect each of these causal processes. $^{89}$

The role of imaging to inform causal models of disease allows the design of rational, precise and optimally powered clinical trials. This can de-risk trials, with better designs and endpoints, including early closure of futile lines of enquiry so as to direct resources more effectively.

This review illustrates the advances in neuroimaging, beyond the structural imaging used clinically every day for diagnosis ${ }^{\mathrm{w} 75-78}$ and staging. ${ }^{10} 11 \mathrm{w} 79-84$ Novel ligands for positron emission tomography (PET) localise and relate molecular processes to each other in vivo. Combining this with connectivity analyses allows the direct evaluation of hypotheses of disease progression. The use of focal neurodegenerative syndromes as disease models allows the delineation of core neuronal systems, which can then be related back to help us understand the complex behavioural abnormalities that arise in dementia. Model-based analyses reveal microcircuit-level consequences of neurodegeneration in experimental medicine studies targeting specific disease mechanisms. With the advent of new therapies, neuroimaging is of greater utility than ever. 


\section{Box 1 Highlights}

- Neuroimaging can be used to establish and test models of disease mechanisms in humans.

- Positron emission tomography can quantify and localise molecular processes in vivo. Amyloid imaging has already changed clinical trials design and identified new drug targets.

- New ligands for synaptic density, protein synthesis, tau and other proteins are scientifically informative but have yet to find their place in healthcare.

- Neuronal populations are functionally and structurally connected at multiple scales, which can be examined by multimodal brain imaging.

- Relating molecular pathology to brain connectivity reveals disease mechanisms and validates drug targets.

- Focal neurodegenerative syndromes are important disease models, selectively perturbing complex neuronal systems.

- Powerful model-based analyses can reveal microcircuit-level consequences of neurodegeneration, in humans.

- Neuroimaging can enrich and stratify cohorts, for precision medicine approaches.

- Small-N experimental medicine studies and large-N observational trials enable the generation and testing of complementary hypotheses.

- Data sharing is now readily available, facilitated by consensus data formats and infrastructure like the Dementias Platform UK Portal, enhancing the value of imaging data through Open Data initiatives, meta-analysis and repurposing. Diseasespecific examples such as Alzheimer's Disease Neuroimaging Initiative and Parkinson Progression Marker Initiative, as well as global initiatives such as ENIGMA have transformed the field of collaborative research.

- Clinical trials can build on the success of longitudinal cohort studies combining behavioural and cognitive assessment with multimodal imaging, genetics, serum and cerebrospinal fluid measures.

\section{Quantifying molecular pathologies}

Current ligands for PET allow the topographical quantification of metabolic activity $\left(\left[{ }^{18} \mathrm{~F}\right]\right.$-fluorodeoxyglucose, FDG), beta amyloid (eg, PiB), tau neuropathology (eg, flortaucipir), neuroinflammation (eg, translocator protein (TSPO) and P2X7R ligands) and synaptic loss (eg, UCB-J).

In clinical practice, FDG reveals changes in regional metabolic activity that differentiate major dementia syndromes. ${ }^{\text {w85 } 86}$ FDGPET has largely superseded single photon emission computed tomography (SPECT) quantification of regional blood flow, due to its greater resolution, signal to noise ratio and robustness to non-linear relationships between metabolic demand and blood flow in cerebrovascular disease. ${ }^{\mathrm{w} 87-89}$ It can also be used as a trial outcome: tracking cerebral metabolic rate. ${ }^{\text {w90 }}$

The synthesis of amyloid ligands (Pittsburgh compound B PiB, florbetapir, florbetaben and flutemetamol) enabled PET quantification of brain beta-amyloid. ${ }^{12}$ In clinical practice it is useful to segregate mild cognitive impairment (MCI) with underlying Alzheimer's pathology from other causes. ${ }^{\text {w91 }} 92$ It can also enrich study populations in clinical trials, screening out patients who are amyloid negative. ${ }^{\text {w93 } 94}$ Amyloid PET has been used as gold standard for validation of biomarkers of Alzheimer's disease (AD) in cerebrospinal fluid (CSF), ${ }^{\text {w95 }}$ and blood. ${ }^{\mathrm{w} 96}{ }^{97}$ However, amyloid burden stabilises by the time of diagnosis, and these ligands have little utility in tracking $\mathrm{AD}$ progression. ${ }^{\mathrm{w} 98-101}$
Longitudinal tracking of disease is more promising from ligands binding Tau. ${ }^{\mathrm{w} 102} 103 \mathrm{Tau}$-aggregates are a defining feature of $\mathrm{AD}$ and FTLD-tau. In AD, the distribution of tau but not beta-amyloid determines phenotype ${ }^{13}$ and progression. ${ }^{\text {w104 }}$ In molecular terms, beta-amyloid promotes the development and propagation of paired helical filaments of tau, which are especially neurotoxic in their oligomeric form. ${ }^{\mathrm{w} 105-107}$ This toxicity is amplified by the presence of beta-amyloid. ${ }^{\mathrm{w} 108}$ Several radioligands have been developed to assess regional Tau burden, including PBB3 ${ }^{\mathrm{w} 109}$ and the THK-series, ${ }^{\text {w110 }}{ }^{\mathrm{b}}$ but the most extensively evaluated is AV-1451, also known as T807 or flortaucipir. ${ }^{\mathrm{w} 111112}$ This ligand has desirable properties in vitro, colocalising in postmortem samples with tau but not beta-amyloid, TDP-43 or $\alpha$-synuclein. ${ }^{\mathrm{w} 113}$ It recapitulates Braak stages of $\mathrm{AD},{ }^{\mathrm{w} 114-116}$ and mirrors the regional distribution in focal subtypes. ${ }^{\mathrm{w} 117} \mathrm{AV}-1451$ binding is more closely linked to hypometabolism, atrophy and cognitive impairment than amyloid-PET.

Outside of $\mathrm{AD}$, tau pathology characterises progressive supranuclear palsy (PSP), ${ }^{\text {w118 }}$ corticobasal degeneration ${ }^{\mathrm{w} 119}$ and half of frontotemporal dementia (FTD). ${ }^{\text {w120 }}{ }^{121}$ Tau is also a modifier of Lewy body disease. ${ }^{\mathrm{w} 122}{ }^{123}$ However, in non-AD diseases, the tau isoforms differ, lacking the characteristic ultrastructure of paired helical filaments in $\mathrm{AD},{ }^{\text {w124-126 }}$ reducing binding affinity. ${ }^{\mathrm{w} 127}$ Despite relative in vitro insensitivity to these alternate isoforms, AV-1451 can identify the distribution of disease in $\mathrm{PSP}^{14}{ }^{14} \mathrm{CBS}^{\mathrm{w} 128}$ and FTD due to mutations of the MAPT gene. ${ }^{\text {w129 }}$ 130

However, AV-1451 also has off-target binding, other than tau. For example, it recapitulates the distribution of pathology in semantic dementia, ${ }^{15}{ }^{16}$ which is characterised by aggregation of TDP-43 in the absence of tau. ${ }^{\text {w131 }} 132$ This lack of specificity is a barrier to stratifying cases of FTD according to molecular aetiology. ${ }^{\mathrm{w} 102}$ There remains an unmet need for specific PET ligands for FTLD-tau, TDP-43 and $\alpha$-synuclein.

Neuroinflammation occurs in many neurodegenerative diseases, with genetic ${ }^{\mathrm{w} 133-135}$ and epidemiological ${ }^{\mathrm{w136}}{ }^{137}$ associations; and postmortem ${ }^{\text {w138 } 139}$ and CSF ${ }^{\mathrm{w} 140} 141$ concomitance; matched in animal models. ${ }^{\text {w142-144 }}$ This underlies an array of PET radiotracers for the neuroinflammatory cascade. ${ }^{17}$ The most established target is the $18 \mathrm{kDa}$ TSPO, which is upregulated in activated microglia (and to some extent astrocytes). Increased microglial activation is seen in vivo in many neurodegenerative diseases, ${ }^{\mathrm{w} 145-150}$ and neuroinflammatory burden correlates with cognition. ${ }^{\text {w151-153 }}$ Neuroinflammation is an early process in pathogenesis, preceding symptom onset in genetic cases, ${ }^{\text {w154 }}$ and persisting through the disease course. ${ }^{\text {w146-148 }}$ Second-generation TSPO ligands have improved signal-to-noise, but interindividual comparisons are confounded by genetic polymorphism. ${ }^{\mathrm{w} 155}$ Novel inflammation-related targets include the P2X7-receptor, ${ }^{\mathrm{w} 156}$ expressed by microglia and a therapeutic target in early AD. ${ }^{\mathrm{w} 157}$

PET ligands for new pathogenic mechanisms are emerging. Changes in synaptic density may precede atrophy and symptom onset, ${ }^{1819 \mathrm{w} 158}$ and can now be quantified by targeting synaptic vesicle glycoprotein $2 \mathrm{~A}$, with ligands such as ${ }^{11} \mathrm{C}-\mathrm{UCB}-\mathrm{J}$. This reveals $20 \%-40 \%$ reductions in regional synaptic density in $\mathrm{AD}$ and non- $\mathrm{AD}$ dementias, in proportion to disease severity. ${ }^{19}$ Postsynaptic pathology can also be measured by PET. For example, TARP $\gamma 8$ regulates surface expression of postsynaptic AMPA receptors. ${ }^{\text {w159 }}$ Ligands targeting TARP $\gamma 8$ are in human use, including early trials. ${ }^{\mathrm{w} 160}{ }^{161}$ Cerebral protein synthesis rates can be measured with L- $\left[1-{ }^{11} \mathrm{C}\right]$ Leucine PET, ${ }^{162} 163$ a technique that has already been applied to children with developmental delay ${ }^{\mathrm{w} 164}$ and young adults with Fragile X syndrome, ${ }^{\mathrm{w} 165}$ and 
holds particular promise for upcoming trials targeting proteostasis and the unfolded protein response. ${ }^{\text {w160 }}$

Advances in PET also derive from new methods of analysis. For example, traditional approaches use mass-univariate 'voxelwise' tests, or comparisons within specified regions of interest. In contrast, one can study the distribution of binding across disease subtypes. This is particularly powerful where binding affinity varies according to molecular pathology, such as in AV-1451's affinity with different mutations of the Tau gene in FTD, ${ }^{\text {w129 }}$ ${ }^{154}$ or neuropathological subtypes of FTD. ${ }^{48} 15 \mathrm{w} 166$ Differences

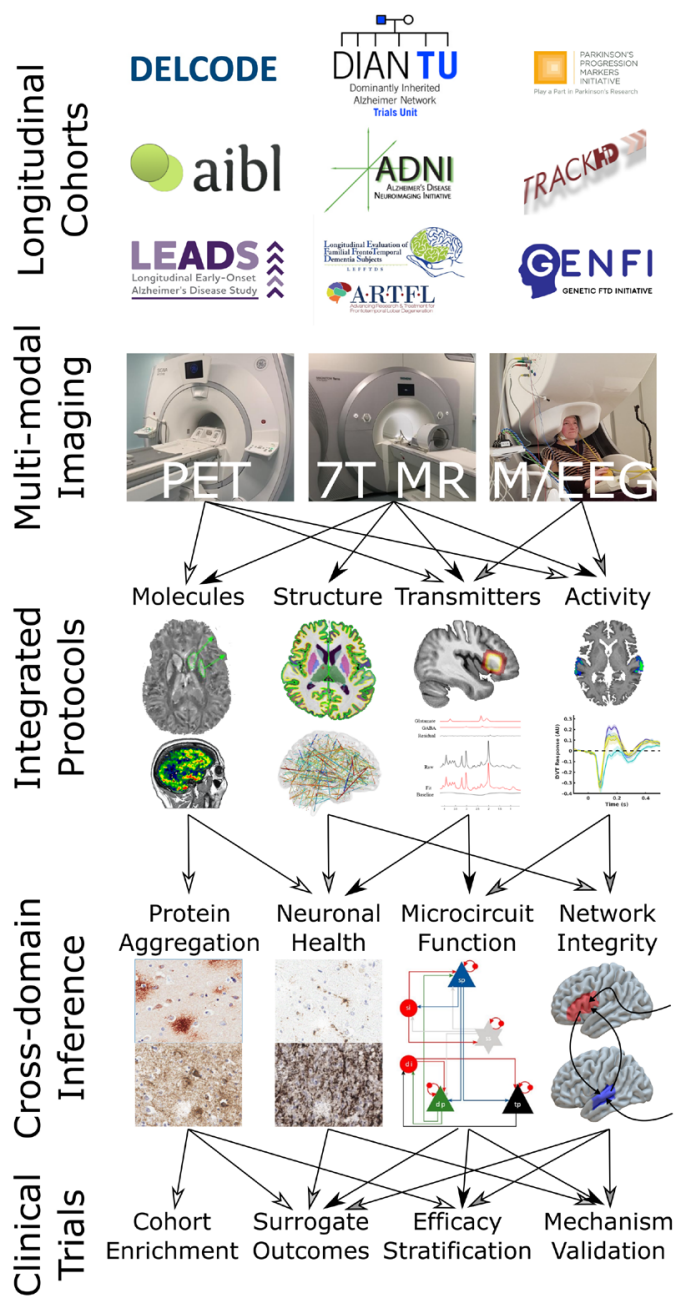

Figure 1 A flow chart illustrating the ways in which carefully designed, multimodal, longitudinal studies can lead to powerful cross-domain inference about neurodegenerative processes. The molecular imaging shown is a 7-tesla QSM (image courtesy of Catarina Rua) ${ }^{\text {w169 }}$ and PK11195 PET. ${ }^{\text {w154 }}$ The structural imaging is a FreeSurfer cortical thickness map from a 7-Tesla MP2RAGE image, above a connectivity map that could be derived from diffusion tractography or resting state functional imaging. The transmitter imaging is 7-Tesla MR Spectroscopy (image courtesy of Murley et al). ${ }^{50}$ The activity images are 7-Tesla auditory fMRI and source-resolved evoked magnetoencephalography responses from superior temporal gyrus. Protein aggregation histopathology images are of amyloid and tau in Alzheimer's disease. Neuronal health histopathology images are of activated microglia and synaptic integrity. The microcircuit shown is that from an extended dynamic causal model. ${ }^{4758}$ The network integrity schematic summarises the inference from coherence and causality techniques for assessing effective connectivity. ${ }^{42}$ fMRI, Functional MRI; PET, positron emission tomography; QSM, Quantitative Susceptibility Mapping. in affinity undermine the contrast between groups for any given region or voxel, but they do not prevent the multivariate approach assessing the similarity of ligand distributions, and classifying individuals into groups based on measures of clustering. This is analogous to the multivoxel pattern analysis techniques used for cognitive decoding in functional MRI (fMRI) ${ }^{20}$ and magnetoencephalography (MEG). ${ }^{\text {w167 }}$

Molecular imaging is no longer solely the preserve of PET. Higher MRI field strengths open up the possibility of richer applications of spectroscopy, with signal to noise ratios sufficient for measurement of GABA in patients. ${ }^{21}$ Quantitative susceptibility mapping ${ }^{\mathrm{w} 168} 169$ assesses the regional burden of paramagnetic substances, with iron particularly linked to cognition in both Alzheimer's ${ }^{22}$ and Parkinson's ${ }^{\text {w170 }}$ disease (PD).

Neurodegenerative diseases are often accompanied by vascular comorbidity, with which they can interact. ${ }^{\text {w171 }} 7 \mathrm{~T}$ highresolution time-of-flight angiography enables the classification of individual hippocampal vascularisation patterns, and support individual assessments of hippocampal vascular reserve. ${ }^{23 \mathrm{w} 172}$ Or, enlarged perivascular spaces may indicate a failure to clear fluid and waste products, ${ }^{\text {w173 }}$ including amyloid and tau. ${ }^{\text {w174-176 }}$ By combining these novel metrics with classical quantifications like white matter intensities and cortical microbleeds, a comprehensive vascular profile can complement molecular, structural and functional imaging for precision medicine.

\section{Multimodal imaging to test disease mechanisms}

Multimodal studies combining PET ligands for metabolism, protein aggregation and neuroinflammation are particularly powerful to examine underlying disease processes. Early studies established the strategy of amyloid PET to confirm underlying Alzheimer's pathology before assessing neuroinflammation. ${ }^{\text {w152 }} 177$ The multimodal approach also reveals associations between neuroinflammation and metabolic impairment, ${ }^{\mathrm{w} 178-180}$ and the colocalisation of neuroinflammation and protein aggregation in $\mathrm{AD}^{24}{ }^{24} \mathrm{PSP}^{\mathrm{w} 181}$ and FTD. ${ }^{8}$ Such combinations can address the relative prognostic value of imaging markers, and clarify the functionally relevant processes to prioritise for disease modifying treatment. ${ }^{25}$ Together these studies demonstrate the interplay between critical disease processes, elucidating the cascade of pathogenic mechanisms.

Molecular imaging can be combined with imaging of neuronal connectivity, including diffusion MRI, fMRI and MEG. Neuronal populations are functionally and structurally connected at a number of scales, from microcircuits within a cortical column ${ }^{\text {w182 }}$ through local, modular connectivity communities ${ }^{\mathrm{w} 183}$ to whole-brain networks. $^{\text {w184 }}$

While there is a high degree of correspondence between structure and function in the healthy brain, the same is not necessarily true in dementia. For example, early synaptic loss and neurotransmitter deficits can alter function without cell death (atrophy). ${ }^{26 \mathrm{w} 185}$ The transition from presymptomatic to symptomatic stages of neurodegeneration is more closely related to a loss of functional connectivity and information transfer in brain networks than a sudden change in structure. ${ }^{27}$ In $\mathrm{AD}$, this is reflected in close associations between tau burden and hippocampal function, irrespective of hippocampal volume, ${ }^{28}$ and a stronger relationship between functional connectivity and memory than with atrophy. ${ }^{\text {w186 }}$ Functional adaption may occur in structurally healthy brain remote from the site of neurodegeneration, ${ }^{29}$ or within areas of early neurodegeneration. ${ }^{\text {w187 }}$

Seemingly inconsistent reports of the relationship between atrophy and hypometabolism are reconciled when considered in terms of networks. For example, while published neuroimaging 
Table 1 Examples of multicentre dementia studies including an advanced imaging component

\begin{tabular}{|c|c|c|c|c|}
\hline Study name & Acronym & No recruited & Participant characteristics & No of sites \\
\hline Alzheimer's Disease Neuroimaging Initiative ${ }^{56}$ & ADNI & $\begin{array}{l}800 \text { (ADNI1)+ } \\
200 \text { (ADNI GO)+ } \\
650 \text { (ADNI2) }\end{array}$ & $\mathrm{AD} / \mathrm{MCl}$, controls & 57 (USA+Canada) \\
\hline \multirow[t]{2}{*}{ Parkinson's Progression Markers Initiative ${ }^{59}$} & \multirow[t]{2}{*}{ PPMI } & 400 & Parkinson's disease & \multirow[t]{2}{*}{30 (12 countries) } \\
\hline & & 200 & Controls & \\
\hline Longitudinal Frontotemporal Lobar Degeneration ${ }^{6 \mathrm{w} 271}$ & ARTFL-LEFFTDS & 1560 & FTD & 14 (USA+Canada) \\
\hline Genetic Frontotemporal dementia Initiative $e^{5}$ & GENFI & 1100 & Familial FTD & 27 (11 countries) \\
\hline Dominantly Inherited Alzheimer Network Trial ${ }^{7 w 72}$ & DIAN-TU & 490 & Familial AD & 42 (17 countries) \\
\hline Australian Imaging, Biomarker \& Lifestyle Flagship Study of Ageing ${ }^{\text {w272 }}$ & AIBL & 1000 & Ageing individuals, $\mathrm{AD} / \mathrm{MCl}$ & 6 (Australia) \\
\hline DZNE Longitudinal Cognitive Impairment and Dementia Study study ${ }^{60}$ & DELCODE & 1000 & Predementia & 8 (Germany) \\
\hline Longitudinal Early-Onset Alzheimer's Disease Study ${ }^{\text {w273 }}$ & LEADS & 600 & $A D$ aged between 40 and 64 & 14 (USA) \\
\hline 4 Repeat Tauopathy Neuroimaging Initiative ${ }^{\text {w274 }}$ & 4RTNI & 110 & PSP or CBD & 2 (USA) \\
\hline $\begin{array}{l}\text { Progressive Supranuclear Palsy Corticobasal Syndrome Multiple System Atrophy } \\
\text { Longitudinal Study }{ }^{\text {275 }}\end{array}$ & PROSPECT & 900 & PSP, CBS, atypical Parkinsonism & 7 (UK) \\
\hline
\end{tabular}

studies of dementia in PD do not show consistent effects when meta-analysed with traditional univariate methods, a network mapping approach revealed consistent dysfunction in a network centred on the hippocampus. ${ }^{30}$ Similar approaches have highlighted the involvement of different connectivity networks across neurodegenerative syndromes. ${ }^{31}$

It has been consistently observed in both structural ${ }^{32}$ and functional $^{\text {w188 }}$ 'connectomes', that brain regions that are most densely connected are most vulnerable to neurodegeneration. The properties of these densely connected 'hubs'w189 can be quantified mathematically with graph theory ${ }^{\mathrm{w} 190}$ or structural equation modelling. ${ }^{\text {w191 }}$ The connectomic approach has yielded novel insights into disease mechanisms in dementia, including vulnerability of long connections in Huntington's disease; ${ }^{\mathrm{w} 192}$ initiation of Alzheimer's ${ }^{33}$ and mechanisms of hallucinations in Parkinson's dementia. $^{34}$

In $\mathrm{AD}$, hub regions may be vulnerable because they are most likely to receive pathological proteins that propagate transneuronally, in a 'prion-like' manner. ${ }^{\text {w193-195 }}$ Much as countries with highly connected airports are more vulnerable to viral epidemics, brain hubs are more likely to receive pathology from 'seed' regions. ${ }^{35-37 w 196} 197$ Combining PET of protein aggregation and functional imaging of brain connectivity using MRI or MEG provides in vivo evidence for this process in humans that was only previously available in animal models, ${ }^{9 \mathrm{w} 198-201}$ probing disease across temporal and spatial dimensions.

Although implicated in neurodegenerative disease, transneuronal spread may not be the only cause of hub vulnerability. Multimodal imaging studies demonstrate that in PD, differential gene expression contributes ${ }^{\mathrm{w} 202}$ while, in PSP, higher metabolic demand $^{38}$ or reduced trophic support ${ }^{\mathrm{w} 203}$ may link protein aggregation and abnormal connectivity. ${ }^{9}$ It is likely that differences in the ultrastructure of the pathological protein affect its propensity to traffic transneuronally, ${ }^{\text {w125 }}$ leading to differences between widespread pathology in network-level diseases such as $\mathrm{AD}$ and motor neuron disease (amyotrophic lateral sclerosis) vs more focal neurodegeneration in PSP and semantic dementia. Similar principles apply to synucleopathies, with different strains in MSA and LBD. ${ }^{\text {w204 }}$

\section{Relating neuronal properties to complex behaviours}

Functional imaging can assess real-time connectivity between regions, with fMRI, M/EEG or direct electrode recordings. These

Table 2 Challenges, possible solutions, limitations and hurdles for neuroimaging to support translational medicine in dementia

\begin{tabular}{|c|c|c|}
\hline Challenge & Solutions & Limitations and hurdles \\
\hline Reproducibility crisis and underused data & $\begin{array}{l}\text { Open data in BIDS format, infrastructure like the Dementias } \\
\text { Platform UK Portal, analysis code publication. }\end{array}$ & Very large file sizes, especially for neurophysiological data. \\
\hline Requirement for large cohorts in clinical trials & $\begin{array}{l}\text { Multisite cooperation through research networks that benefit all } \\
\text { collaborators. }\end{array}$ & $\begin{array}{l}\text { Funding climates that prioritise competitive over collaborative } \\
\text { research. }\end{array}$ \\
\hline $\begin{array}{l}\text { Limited clinical, research and imaging resources to } \\
\text { undertake deep and multimodal phenotyping }\end{array}$ & $\begin{array}{l}\text { Standardised protocols. Collaborative grant funding. Cross- } \\
\text { scanner harmonisation efforts like the UK7T partnership. }\end{array}$ & $\begin{array}{l}\text { Differing research goals between centres. Locally optimal } \\
\text { protocols may not be maximally transferrable. }\end{array}$ \\
\hline Non-specific binding of PET ligands & $\begin{array}{l}\text { Studies across comprehensive patient cohorts, multivariate } \\
\text { pattern-analysis methods, neuropathological validation. }\end{array}$ & $\begin{array}{l}\text { Large no of ligands from different vendors makes } \\
\text { comprehensive characterisation challenging. }\end{array}$ \\
\hline Slow translation to the clinic & $\begin{array}{l}\text { Interdisciplinary work, collaboration with pharmaceutical } \\
\text { companies in the design phase. }\end{array}$ & $\begin{array}{l}\text { Inertia and a preference for insensitive but established } \\
\text { measures in trial design and regulatory approval. }\end{array}$ \\
\hline $\begin{array}{l}\text { A proliferation of measures with unclear relative sensitivity } \\
\text { and predictive value. }\end{array}$ & Head-to-head comparison studies in the same participants. & $\begin{array}{l}\text { Studies are expensive, and rely on clinical tests that may be } \\
\text { insensitive. }\end{array}$ \\
\hline $\begin{array}{l}\text { Large-scale clinical trials are hugely expensive, and not all } \\
\text { mechanisms can be explored. }\end{array}$ & $\begin{array}{l}\text { Small-n pharma coimaging studies to demonstrate proof } \\
\text { of concept, motivating larger trials by rescuing functional } \\
\text { biomarkers. }\end{array}$ & $\begin{array}{l}\text { Rescuing imaging biomarkers and restoring neurotransmitter } \\
\text { balance may be insufficient to provide clinical efficacy. }\end{array}$ \\
\hline $\begin{array}{l}\text { Individual variation within patient cohorts can mask real } \\
\text { effects. }\end{array}$ & $\begin{array}{l}\text { Multimodal neuroimaging to provide post hoc explanations of } \\
\text { subgroup efficacy, leading to personalised medicine. }\end{array}$ & $\begin{array}{l}\text { Prespecifying such analyses is often difficult, and false-positive } \\
\text { associations mean insights become less trustworthy and } \\
\text { generalisable. }\end{array}$ \\
\hline $\begin{array}{l}\text { Big data become increasingly difficult to analyse, and } \\
\text { statistically significant findings can have small effect sizes or } \\
\text { be driven by hidden bias. }\end{array}$ & $\begin{array}{l}\text { Formal statistician involvement. Prespecified analyses. Artificial } \\
\text { intelligence and machine learning techniques. }\end{array}$ & $\begin{array}{l}\text { As algorithms become more complex, they can become less } \\
\text { transparent and interpretable for patients and clinicians. }\end{array}$ \\
\hline
\end{tabular}

BIDS, brain imaging data structure; PET, positron emission tomography. 
methods fall into two broad categories ${ }^{39}$ : (1) 'functional connectivity' describes activity between brain regions that is correlated over time or phase coherent, reprising Hebb's principle that neurons that are wired together fire together and (2) 'effective connectivity' refers to directional influences with one region causing change in another; assessed by techniques such as dynamic causal modelling ${ }^{\text {w205 }}$ or Granger causality. ${ }^{\text {w206 }}$

In the healthy brain, the structure of connections is closely matched to the strength of their functional connectivity, set within overall cortical connectivity gradients ${ }^{40}$ over a range of spatial scales. $^{\text {w207 }}$ This applies at rest (the so-called 'resting state') and during tasks. ${ }^{\mathrm{w} 208}{ }^{209}$ However, the structure-function homology breaks down in dementia. The strength of resting-state connectivity between two brain regions typically falls as they are affected by neurodegeneration. ${ }^{41}$ However, when those regions are engaged by a task, their connectivity may paradoxically increase, perhaps in compensation as they 'work harder' to perform a cognitive operation with a less efficient neural architecture. ${ }^{42}$ Changes in structurefunction coupling can also be measured using an approach that considers the gradients of hierarchical organisational, ${ }^{40}$ which then show decoupling in neurodegenerative diseases such as Parkinson's. ${ }^{\text {w212 }}$

To explain how biological, psychological and social variables contribute to the overall expression of disease, Watershed models ${ }^{\mathrm{w} 213}$ use the analogy of a river. Intermediate stages of the hierarchy are termed 'endophenotypes', which can be observed by cognitive or neural properties. Such modelling of multi-modal neuroimaging can explain complex traits, ${ }^{43}$ and give insights into the factors that mediate epidemiological observations; for the association between mid-life obesity and late-life neurodegeneration is accounted for by changes in white matter integrity. ${ }^{\mathrm{w} 214}$ These models can be extended by the addition of a phenotypic 'delta' at the end of the metaphorical river; recognising that multiple cognitive and behavioural outputs stem from different admixtures of common neuronal quantities. Multivariate analysis of large cohorts ${ }^{\mathrm{w} 215}$ can relate imaging and cognitive parameters, and describe how this relationship changes with age. ${ }^{\mathrm{w} 216217}$

The way in which age and disease moderates the relationship between structure, function and behaviour is not linear. For example, in MCI, hippocampal or entorhinal atrophy results in hyperactivation of medial temporal lobe circuitry. ${ }^{\text {w186 }} 218$ Such local hyperactivity may act in a vicious circle to promote local amyloid deposition. ${ }^{44}$ This may be a more general property of neurodegenerative disease, as highly active 'hubs' initially compensate for declines in structural connectivity by increasing their firing rate. ${ }^{\mathrm{w} 219}$ Later in disease this compensation breaks down. ${ }^{45}$ Cross-sectional studies relating neuronal connectivity to pathological protein deposition have been crucial to corroborating these hypotheses, ${ }^{9}$ and will be strengthened in future by large, longitudinal cohort studies employing multimodal imaging, allowing generation and testing of prevention and treatment hypotheses.

\section{Assessing microcircuit-levels in humans}

So far, we have discussed the macroscopic level of brain structure and function, representing large neuronal populations and the major white-matter connections between them. We turn next to the mesoscopic- and microscopic levels, where initiating events in the neurodegenerative cascade occur. ${ }^{\mathrm{w} 61-63}$

Ultrahigh resolution structural imaging with 7-Tesla MRI is sensitive to functional and volumetric changes on the order of hundreds of microns (cf. a grain of sand). However, these field strengths are particularly sensitive to susceptibility changes, and as a result are able to detect microbleeds ${ }^{\mathrm{w} 220}$ and iron-dense amyloid plaques that are invisible with standard hospital imaging. ${ }^{\text {w221 }}$ Recent advances in laminar fMRI have opened up the ability to examine the functional consequences of degenerative changes within specific cortical layers. ${ }^{46 w 222-224}$

Synapse loss occurs early in AD, ${ }^{\text {w225 }}$ perhaps due to direct synaptotoxicity of beta-amyloid and tau aggregates. ${ }^{26 \mathrm{w} 226-229}$ This is reflected in functional imaging, for example by taurelated reductions in fMRI hippocampal novelty responses, ${ }^{28}$ amyloid-related resting state alterations, ${ }^{\text {w230 }}$ and inflammation related changed in connectivity of the medial temporal lobe. ${ }^{\text {w231 }}$ Amyloid pathology can also be associated with intrinsic neuronal hyperexcitability ${ }^{\mathrm{w} 232}$ and inhibitory dysfunction. ${ }^{47 \mathrm{w} 233}$

The temporal resolution of MEG and EEG (electroencephalography) provide an opportunity to assess the neurophysiological signatures of neurodegenerative diseases, especially in terms of oscillatory dynamics. Neurodegenerative diseases are commonly classified by location of pathology, which tends to track phenotype, but they also cause distinctive changes in the temporal structure of neuronal communication, both at rest ${ }^{48}$ and when engaged in a task. ${ }^{\mathrm{w} 234}$ These changes can support disease classification even between patients in whom the localisation of pathology is similar, providing a first step towards precision medicine when therapies become available for specific proteinopathies.

Reaching for the microscopic scale with neuroimaging is a challenge, but detailed biophysical models can now be built, to examine the mechanisms of neurodegeneration. For example, dynamic causal modelling ${ }^{\mathrm{w} 235}$ can be applied to MEG/EEG ${ }^{\mathrm{w} 236}$ and $\mathrm{fMRI}^{\mathrm{w} 205}$ data, to infer the state of laminar-specific cellular populations and synaptic dynamics. ${ }^{\mathrm{w} 182} 237$ When combined with pharmacological intervention, these techniques can assess dynamics that are specific to individual neurotransmitters and receptor populations, allowing a precision of in vivo therapeutic assessment that was hitherto only possible with preclinical models of dementia.

\section{Experimental medicine studies}

Neuroimaging can enhance experimental medicine studies in several ways.

First, imaging biomarkers can identify presymptomatic cases. Early intervention may be more effective to reduce the long-term burden of disease, or even prevent symptom onset. For example, amyloid PET imaging is commonly used to identify those with Alzheimer pathology, either as latent disease in presymptomatic individuals, or to confirm AD pathology in MCI. ${ }^{\text {w238 } 239}$ There is a $3 \%$ annual risk of conversion to amyloid positivity in cognitively normal people over 65 , increasing to $7 \%$ if positive for apolipoprotein $\varepsilon 4 .{ }^{\mathrm{w} 240}$ These individuals show more rapid cognitive decline than amyloid-negative peers. ${ }^{\mathrm{w} 241}$ This can enrich clinical trials. However, screening for Alzheimer's pathology is problematic: elderly amyloid-positive cognitively healthy individuals still only have an $11 \%$ annual risk of conversion to MCI or $\mathrm{AD},{ }^{\mathrm{w} 242}$ meaning that trials enriched in this way could still take years. Tau imaging with flortaucipir may stratify such cohorts further. Moving forwards, such imaging biomarkers will be increasingly important to identify presymptomatic pathology in those at risk of sporadic or genetically determined dementias, whether $\mathrm{AD}^{7 \mathrm{w} 72}$ or FTD. ${ }^{56}$

Second, neuroimaging provides an array of surrogate outcome measures that can change more quickly and be quantified more precisely than behavioural and cognitive measures. While rescuing imaging biomarkers is not sufficient, it can provide evidence of a treatment's effect on the brain as a prelude to clinical endpoints in later phase trials. ${ }^{\mathrm{w} 243}$ Atrophy is most 
widely used in this way, the logic being that a treatment that slows atrophy has influenced neuronal survival, and is more likely to be clinically effective. ${ }^{\text {w244 } 245}$ Ultrahigh field imaging (7-Tesla MRI) increases the anatomical resolution and neurochemical sensitivity of MRI, 3-5 x compared with 3T MRI. ${ }^{\text {2246 }}$ Hippocampal subfields can be imaged with a resolution of a few hundred microns. ${ }^{\text {w247 }}$

However, atrophy resulting from extensive cell death is a late process in the pathogenesis of dementias. Upstream events may be more suitable for earlier intervention, such as the loss of synapses $^{18} 19$ and inflammation. ${ }^{82}$ To test the relative performance of imaging biomarkers, and against fluidic biomarkers and clinical rating scales, requires a head-to-head comparison. For this, the Deep and Frequent Phenotyping study ${ }^{\text {w248 }}$ is underway, to compare established and novel metrics of the progression of Alzheimer pathology. fMRI, multiligand PET and MEG are longitudinally assessed alongside behavioural measures, CSF, blood and saliva biomarkers.

Third, neuroimaging can test candidates for restoration of the neural mechanisms of aberrant behaviour in small-n studies, over short timescales. For example, MEG demonstrates that frontal lobe neurophysiological signatures of behavioural inhibition are reduced in behavioural variant FTD and partially recovered by correction of the serotonergic deficit in FTD. ${ }^{49 w 249-250}$ No change was demonstrated in behaviour, either because of power or because, using the watershed analogy described above, serotonergic deficiency is only one tributary to the river of behavioural disinhibition in FTD, alongside atrophy, loss of frontal oscillatory connectivity ${ }^{48 \mathrm{w} 234}$ and GABA-ergic depletion. ${ }^{2150}$

Fourth, by identifying causes of heterogeneity, neuroimaging methods enable cohort enrichment and stratification at inclusion; and can provide post hoc explanations of variation in a treatment response. For example, the selective norepinephrine reuptake inhibitor atomoxetine was unsuccessful in rescuing response inhibition in PD, but subgroup analysis revealed that the drug was effective in those with more severe disease, and intact fronto-striatal connectivity. ${ }^{\text {w251 }}$ Preserved cortical outflow tracts were proposed as necessary for behavioural function to be improved following functional restoration 'upstream' in prefrontal cortex. ${ }^{\text {w252 } 253}$ Such analyses of multivariate data can reveal effects within heterogeneous populations, and develop protocols to predict individual responses to medication, to realise personalised medicine. ${ }^{51}$ The heterogeneity of a cohort can be formally dissected according to stage (severity) and/or phenotype. For example, the SuStaIn model uses machine learning to distinguish subtypes from progression of disease, against which treatment efficacy could be individually assessed. ${ }^{52}$

Fifth, neuroimaging with novel PET ligands can be applied in proof of concept and dose finding. For example, inhibition of O-GlcNAcase reduces phosphorylation of Tau in mice, ${ }^{\text {w254 }}$ and has been proposed as a therapeutic strategy in $\mathrm{AD}$ and PSP. ASN120290 is a novel, orally delivered inhibitor of this enzyme that has completed phase 1 safety trials. ${ }^{\mathrm{w} 255}$ In advance of a phase 2 trial, a dose-finding study uses PET to image enzymatic function in vivo. ${ }^{\mathrm{w} 256}$ This novel approach may reduce the number of participants needed in phase 2 trials, a crucial advantage when dealing with rare diseases. Similarly, L-[1- $\left.{ }^{11} \mathrm{C}\right]-$ Leucine PET allows in vivo quantification of modulation of the unfolded protein response in upcoming trials. ${ }^{\text {w160 }}$

Finally, neuroimaging can add value to clinical trials of investigational medicines, to inform our understanding of disease processes, establishing a 'positive feedback' loop in translational research. Early and late phase studies can be designed in a way that includes longitudinal follow-up of large disease cohorts with both established and novel biomarkers. ${ }^{7}$ The benefit of these approaches is increased by data sharing.

\section{Big data and artificial intelligence}

As computing capabilities expand, the concept of 'big data' evolves, but it generally describes information too large or complex to be analysed in traditional ways. Artificial intelligence (AI) and machine learning techniques applied to large, multimodal datasets hold promise for the discovery of complex, non-linear relationships between pathological and environmental factors, improving diagnosis, prevention and treatment. However, with larger-scale data one must be careful to minimise the risks of false discovery through statistical chance or hidden biases, ${ }^{53 \mathrm{w} 257}$ guard against overinterpretating small effect sizes $^{\mathrm{w} 258}$ and maintain interpretability. ${ }^{\text {w259 }}$

Big data may arise from a coordinated effort to obtain a standard set of measures, or to repurpose data generated for other purposes such as healthcare. The former is exemplified by the Human Connectome Project, with 4 hours of imaging in 1200 young volunteers, ${ }^{\text {w260 }}$ and similar acquisitions in 1200 older individuals, 1350 teens, 500 babies/toddlers and 1500 fetuses. ${ }^{\mathrm{w} 261} \mathrm{~A}$ second example is the UK Biobank, which aims to acquire MRI in 100000 individuals for combination with lifestyle, biomarker and genomic data. ${ }^{54}$ Around 40000 scans have been acquired to date.

Big data can also be built by fusion of smaller studies. Data sharing is now readily available, facilitated by infrastructure like the Dementias Platform UK Portal, ${ }^{\text {w262 }}$ enabling researchers to deposit and access imaging and behavioural measures at scale. This facilitates replication, and generation of very largecross-cohort analyses. Consensus data formats such as brain imaging data structure, initially developed for $\mathrm{MRI}^{\mathrm{w} 263}$ and now extended to $\mathrm{MEG}^{\mathrm{w} 264}$ make this process easier. Consortium efforts accounting for differences between scanner and acquisition protocols such as ENIGMA are increasing our ability to pool inference across cohorts, allowing meta-analysis and repurposing. ${ }^{\text {w265 }}$

Big data hold particular promise for hypothesis generation, linked to bespoke studies for hypothesis testing. This reduces the risk of recruitment bias resulting in very large normative cohorts. For example, one must consider whether those with predementia are equally likely to volunteer for a cohort study? Their numbers might either be reduced by latent cognitive difficulties or apathy, or increased by a wish to understand subjective cognitive complaints. ${ }^{\text {w266 }}$

The simplest approach to the analysis of big data is the application of a standardised univariate method at large scale. However, critical effects may be multivariate, complex and non-linear. Assessing complex effects is better suited to methods collectively known as AI. This umbrella term describes a range of approaches from supervised machine learning classification algorithms like support vector machines, ${ }^{\text {w267 }}$ through to 'deep learning' efforts that apply multiple, increasingly abstract, processing layers. ${ }^{55}$ Through such brute force associative techniques, deep learning can achieve very high diagnostic accuracies for dementia. ${ }^{\text {w268 }}$ However, these accuracies are vulnerable to over-fitting, and may lack insight into which disease mechanisms are driving classification. They may fail if the classifier comes across variants not previously observed.

The application of big data approaches to dementia also raises ethical considerations, beyond those of privacy, data security and governance common to all healthcare datasets. ${ }^{\text {w269 }} 270$ First, it may require a change in mindset among clinical specialists, with 
hard-earned diagnostic acumen, to trust algorithms and methods that rely on interactions and hidden states that are not transparent. Second, the responsibility for inaccurate diagnosis would be unclear, a problem likely to be particularly challenging for rarer dementia syndromes. Third, the social, financial and psychological consequences of making a predementia diagnosis in the absence of diseasemodifying treatment are difficult to quantify. The response to these challenges requires consultation beyond the neuroimaging community, maintaining a clear distinction between applications for research and direct patient care.

\section{A roadmap to clinical trials}

We are entering an exciting phase in dementia research, moving from observational to therapeutic studies, with enhanced ability to perform mechanistic analysis of human dementia pathogenesis. Many novel targets have been identified and diverse diseasemodifying agents are coming to clinical trials. Although the ultimate goal is to prevent cognitive and behavioural decline in a way that is meaningful for patients and families, and with health economic benefits, there is an intermediate stage of experimental medicine that will need to exploit quantitative imaging of neuronal and physiological function (figure 1).

There are large, observational, longitudinal cohort studies with multimodal assessment that serve as foundational data on natural history and biomarkers to inform evidence-based interventional trial design. The AD Neuroimaging Initiative changed the landscape by bringing together clinical, imaging and fluid biomarkers to feed into clinical trials. This prototypical study is now in its third phase. ${ }^{56}$ It sets the stage for many collaborative international studies involving advanced imaging, clinical cognitive and fluid biomarkers, summarised in table 1 .

As well as their multimodal imaging data, the open science framework of these initiatives encourages collaboration, and replication and validation of findings. At an even larger scale, the ENIGMA consortium spans diseases and facilitates mega-scale imaging/genetic collaborations to answer mechanistic questions only possible with very large numbers of patients. ${ }^{57}$

Complementary to these initiatives, observational cohorts are critical to deeply phenotype patients, using multimodal imaging and fluid markers. These enable bespoke, experimental and innovative elements to be included and often feed into larger consortia. For example, TRACK-HD ${ }^{\mathrm{w} 73}$ and TRACKON-HD ${ }^{\mathrm{w} 74}$ are multisite observational cohort studies of Huntington's disease. Alongside clinical and fluid biomarkers, neuroimaging data include structural and fMRI. Crucially, clinical trials of antisense oligonucleotide designed to reduce mutant huntingtin protein are underway ${ }^{\mathrm{w} 276}$ and neuroimaging metrics refined in observational cohorts inform long term trials of these and similar agents. Similar comprehensive multimodal observational cohorts have been established across neurodegeneration: in PD, the Vision in Parkinson's study collates retinal, visual, fluid and genetic markers with advanced imaging ${ }^{\text {w277 }} 278$ with the aim of refining markers of progression in PD to stratify patients for clinical trials.

This approach emphasises the value of early-stage feasibility trials to produce meaningful insights into disease mechanisms, paving the way for larger trials. For example, the AZA-PD study $^{\mathrm{w} 279}$ is an early phase double-blind placebo-controlled trial of immunosuppression in PD, aiming to modify progression by reducing neuroinflammation. Azathioprine exerts its effect slowly, and it is anticipated that efficacy as measured by traditional motor scores will be weak over 18 months. However, longitudinal PET imaging of neuroinflammation may provide proof of concept for the therapy.
Overall, intelligent design of the neuroimaging component of trials enables true proof of concept, illustrating feasibility and derisking the process by providing early surrogate endpoints that enable $\mathrm{Go} / \mathrm{Nogo}$ decisions to longer-term cognitive and behavioural endpoints. Medical, scientific, and commercial considerations are complementary, but each enhanced by neuroimaging to work towards a cure for dementia. There are a series of challenges, potential solutions, limitations and hurdles to this overarching goal, summarised in table 2 .

\section{Summary}

Advances in neuroimaging will facilitate the transition from discovery science and drug discovery through to effective and timely clinical trials for dementia and neurodegeneration. We have illustrated the multifaceted roles of PET, MRI and neurophysiology, linked to disease models and AI analysis methods. Understanding of the causes of heterogeneity can be applied to stratify clinical trials, and to the realisation of precision medicine. Building on cross-sector collaboration and best practices for open science, advanced in brain imaging will enhance good clinical care and accelerate dementia prevention and treatment.

see web appenddix for additional references for online supplemental file 1 .

\section{Author affiliations \\ ${ }^{1}$ Department of Clinical Neurosciences, University of Cambridge, Cambridge, UK \\ ${ }^{2}$ MRC Cognition and Brain Sciences Unit, Cambridge, UK \\ ${ }^{3}$ Cambridge University Hospitals NHS Foundation Trust, Cambridge, UK \\ ${ }^{4}$ Dementia Research Centre, University College London, London, UK \\ ${ }^{5}$ National Hospital for Neurology \& Neurosurgery, Queen square, London, UK \\ ${ }^{6}$ Wellcome Centre for Human Neuroimaging, University College London, London, UK \\ ${ }^{7}$ Movement Disorders Centre, University College London, London, UK \\ ${ }^{8}$ Otto-von-Guericke-University Magdeburg Institute of Cognitive Neurology and \\ Dementia Research, Magdeburg, Sachsen-Anhalt, Germany \\ ${ }^{9}$ German Centre for Neurodegenerative Diseases (DZNE), Magdeburg, Germany \\ ${ }^{10}$ Center for Behavioral Brain Sciences (CBBS), Magdeburg, Germany \\ ${ }^{11}$ Institute of Cognitive Neuroscience, University College London, London, UK \\ ${ }^{12}$ Athinoula A. Martinos Center for Biomedical Imaging, Massachusetts General \\ Hospital and Harvard Medical School, Charlestown, Massachusetts, USA \\ ${ }^{13}$ Frontotemporal Disorders Unit, Department of Neurology, Massachusetts General \\ Hospital and Harvard Medical School, Charlestown, Massachusetts, USA}

Twitter Thomas Edmund Cope @tccambs, Rimona Sharon Weil @rimonaweil and James Benedict Rowe @CambridgeFTD

Contributors Writing—original draft: TEC. Writing—review and editing: RSW, ED, $B C D$ and JBR. Visualisation: TEC.

Funding TEC is supported by an NIHR Clinical Lectureship. RSW is funded by a Wellcome Trust Clinical Research Career Development Fellowship (201567/Z/16/Z). JBR is supported by the Wellcome Trust (103838) and MRC (SUAG051/G101400).

Competing interests RSW has received speaker fees from GE Healthcare. BCD has received personal fees from Arkuda, Eli Lilly, Merck, Wave Lifesciences, Novartis, Oxford University Press, Cambrige University Press, and Elsevier; grants and personal fees from Biogen; and grants from the National Institute on Aging, National Institute of Mental Health, and National Institute of Neurological Disorders and Stroke. JBR has performed consultancies with Biogen, UCB, Asceneuron, WAVE, Astex, SVHealth; has received research grants from AZ-Medimmune, Lilly, Janssen; and is an editor at Brain.

Patient consent for publication Not required.

Provenance and peer review Commissioned; externally peer reviewed.

Supplemental material This content has been supplied by the author(s). It has not been vetted by BMJ Publishing Group Limited (BMJ) and may not have been peer-reviewed. Any opinions or recommendations discussed are solely those of the author(s) and are not endorsed by BMJ. BMJ disclaims all liability and responsibility arising from any reliance placed on the content. Where the content includes any translated material, BMJ does not warrant the accuracy and reliability of the translations (including but not limited to local regulations, clinical guidelines, terminology, drug names and drug dosages), and is not responsible for any error and/or omissions arising from translation and adaptation or otherwise. 
ORCID iDs

Thomas Edmund Cope http://orcid.org/0000-0002-4751-1786

Rimona Sharon Weil http://orcid.org/0000-0002-5092-6325

James Benedict Rowe http://orcid.org/0000-0001-7216-8679

\section{REFERENCES}

1 Lee VM-Y, Goedert M, Trojanowski JQ. Neurodegenerative tauopathies. Annu Rev Neurosci 2001;24:1121-59.

2 Insel T, Cuthbert B, Garvey M, et al. Research domain criteria (RDoC): toward a new classification framework for research on mental disorders. Am Psychiatric Assoc 2010.

3 Murley AG, Coyle-Gilchrist I, Rouse MA, et al. Redefining the multidimensional clinical phenotypes of frontotemporal lobar degeneration syndromes. Brain 2020;143:1555-71.

4 Höglinger GU, Respondek G, Stamelou M, et al. Clinical diagnosis of progressive supranuclear palsy: the movement disorder Society criteria. Mov Disord 2017.

5 Rohrer JD, Nicholas JM, Cash DM, et al. Presymptomatic cognitive and neuroanatomical changes in genetic frontotemporal dementia in the genetic frontotemporal dementia initiative (GENFI) study: a cross-sectional analysis. Lancet Neurol 2015;14:253-62

6 Rosen HJ, Boeve BF, Boxer AL. Tracking disease progression in familial and sporadic frontotemporal lobar degeneration: recent findings from ARTFL and LEFFTDS. Alzheimers Dement 2020;16:71-8

7 Bateman RJ, Benzinger TL, Berry S, et al. The DIAN-TU next generation Alzheimer's prevention trial: adaptive design and disease progression model. Alzheimers Dement 2017:13:8-19

8 Bevan-Jones WR, Cope TE, Jones PS, et al. Neuroinflammation and protein aggregation co-localize across the frontotemporal dementia spectrum. Brain 2020;143:1010-26.

9 Cope TE, Rittman T, Borchert RJ, et al. Tau burden and the functional connectome in Alzheimer's disease and progressive supranuclear palsy. Brain 2018;141:550-67.

10 Smith AD. Imaging the progression of Alzheimer pathology through the brain. Proc Natl Acad Sci U S A 2002;99:4135-7.

11 Broe M, Hodges JR, Schofield E, et al. Staging disease severity in pathologically confirmed cases of frontotemporal dementia. Neurology 2003:60:1005-11.

12 Landau SM, Thomas BA, Thurfjell L, et al. Amyloid PET imaging in Alzheimer's disease: a comparison of three radiotracers. Eur J Nucl Med Mol Imaging 2014;41:1398-407.

13 Ossenkoppele R, Schonhaut DR, Baker SL, et al. Tau, amyloid, and hypometabolism in a patient with posterior cortical atrophy. Ann Neurol 2015:77:338-42.

14 Passamonti L, Vázquez Rodríguez P, Hong YT, et al. 18F-AV-1451 positron emission tomography in Alzheimer's disease and progressive supranuclear palsy. Brain 2017:140:781-91.

15 Bevan-Jones WR, Cope TE, Jones PS, et al. [18F]AV-1451 binding in vivo mirrors the expected distribution of TDP-43 pathology in the semantic variant of primary progressive aphasia. J Neurol Neurosurg Psychiatry 2018;89:1032-7.

16 Makaretz SJ, Quimby M, Collins J, et al. Flortaucipir tau PET imaging in semantic variant primary progressive aphasia. J Neurol Neurosurg Psychiatry 2018:89:1024-31

17 Narayanaswami V, Dahl K, Bernard-Gauthier V, et al. Emerging PET radiotracers and targets for imaging of neuroinflammation in neurodegenerative diseases: outlook beyond TSPO. Mol Imaging 2018;17:1536012118792317

18 Chen M-K, Mecca AP, Naganawa M, et al. Assessing synaptic density in Alzheimer disease with synaptic vesicle glycoprotein $2 A$ positron emission tomographic imaging JAMA Neurol 2018;75:1215-24.

19 Holland N, Jones PS, Savulich G, et al. Synaptic Loss in Primary Tauopathies Revealed by [11C]UCB-J Positron Emission Tomography. Mov Disord 2020;35:1834-42.

20 Kriegeskorte N, Mur M, Bandettini P. Representational similarity analysis - connecting the branches of systems neuroscience. Front Syst Neurosci 2008;2:4.

21 Murley AG, Rowe JB. Neurotransmitter deficits from frontotemporal lobar degeneration. Brain 2018;141:1263-85.

22 Ayton S, Fazlollahi A, Bourgeat $P$, et al. Cerebral quantitative susceptibility mapping predicts amyloid- $\beta$-related cognitive decline. Brain 2017;140:2112-9.

23 Perosa V, Priester A, Ziegler G, et al. Hippocampal vascular reserve associated with cognitive performance and hippocampal volume. Brain 2020;143:622-34.

24 Dani M, Wood M, Mizoguchi R, et al. Microglial activation correlates in vivo with both tau and amyloid in Alzheimer's disease. Brain 2018:141:2740-54.

25 Malpetti M, Kievit RA, Passamonti L, et al. Microglial activation and tau burden predict cognitive decline in Alzheimer's disease. Brain 2020;143:1588-602.

26 Hoover BR, Reed MN, Su J, et al. Tau mislocalization to dendritic spines mediates synaptic dysfunction independently of neurodegeneration. Neuron 2010:68:1067-81.

27 Rittman T, Borchert R, Jones S, et al. Functional network resilience to pathology in presymptomatic genetic frontotemporal dementia. Neurobiol Aging 2019;77:169-77.

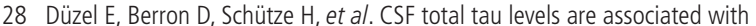
hippocampal novelty irrespective of hippocampal volume. Alzheimers Dement 2018:10:782-90.
29 Cope TE, Shtyrov Y, MacGregor LJ, et al. Anterior temporal lobe is necessary for efficient lateralised processing of spoken word identity. Cortex 2020;126:107-18.

30 Weil RS, Hsu JK, Darby RR, et al. Neuroimaging in Parkinson's disease dementia: connecting the dots. Brain Commun 2019;1:fcz006.

31 Darby RR, Joutsa J, Fox MD. Network localization of heterogeneous neuroimaging findings. Brain 2019;142:70-9.

32 Crossley NA, Mechelli A, Scott J, et al. The hubs of the human connectome are generally implicated in the anatomy of brain disorders. Brain 2014;137:2382-95.

33 Pascoal TA, Mathotaarachchi S, Kang MS, et al. A $\beta$-induced vulnerability propagates via the brain's default mode network. Nat Commun 2019:10:1-13.

34 Zarkali A, McColgan P, Ryten M, et al. Differences in network controllability and regional gene expression underlie hallucinations in Parkinson's disease. Brain 2020:143:3435-48.

35 Zhou J, Gennatas ED, Kramer JH, et al. Predicting regional neurodegeneration from the healthy brain functional connectome. Neuron 2012;73:1216-27.

36 Raj A, Kuceyeski A, Weiner M. A network diffusion model of disease progression in dementia. Neuron 2012;73:1204-15.

37 Raj A, LoCastro E, Kuceyeski A, et al. Network diffusion model of progression predicts longitudinal patterns of atrophy and metabolism in Alzheimer's disease. Cell Rep 2015;10:359-69.

38 Saxena S, Caroni P. Selective neuronal vulnerability in neurodegenerative diseases: from stressor thresholds to degeneration. Neuron 2011;71:35-48.

39 Friston KJ. Functional and effective connectivity: a review. Brain Connect 2011;1:13-36.

40 Margulies DS, Ghosh SS, Goulas A, et al. Situating the default-mode network along a principal gradient of macroscale cortical organization. Proc Natl Acad Sci U SA 2016;113:12574-9.

41 Stam CJ. Modern network science of neurological disorders. Nat Rev Neurosci 2014; 15:683-95

42 Cope TE, Sohoglu E, Sedley W, et al. Evidence for causal top-down frontal contributions to predictive processes in speech perception. Nat Commun 2017;8:2154.

43 Kievit RA, Davis SW, Griffiths J, et al. A watershed model of individual differences in fluid intelligence. Neuropsychologia 2016;91:186-98

44 de Haan W, Mott K, van Straaten ECW, et al. Activity dependent degeneration explains hub vulnerability in Alzheimer's disease. PLoS Comput Biol 2012;8:e1002582.

45 Jones DT, Knopman DS, Gunter JL, et al. Cascading network failure across the Alzheimer's disease spectrum. Brain 2016;139:547-62.

46 McColgan P, Joubert J, Tabrizi SJ, et al. The human motor cortex microcircuit: insights for neurodegenerative disease. Nat Rev Neurosci 2020;21:401-15.

47 Adams NE, Hughes LE, Rouse MA. GABAergic cortical network physiology in frontotemporallobar degeneration. Brain

48 Sami S, Williams N, Hughes LE, et al. Neurophysiological signatures of Alzheimer's disease and frontotemporal lobar degeneration: pathology versus phenotype. Brain 2018; 141:2500-10.

49 Hughes LE, Rittman T, Regenthal R, et al. Improving response inhibition systems in frontotemporal dementia with citalopram. Brain 2015;138:1961-75.

50 Murley AG, Rouse MA, Jones PS, et al. GABA and glutamate deficits from frontotemporal lobar degeneration are associated with disinhibition. Brain 2020;143:3449-62.

51 Ye Z, Rae CL, Nombela C, et al. Predicting beneficial effects of atomoxetine and citalopram on response inhibition in Parkinson's disease with clinical and neuroimaging measures. Hum Brain Mapp 2016;37:1026-37.

52 Young AL, Marinescu RV, Oxtoby NP, et al. Uncovering the heterogeneity and temporal complexity of neurodegenerative diseases with subtype and stage inference. Nat Commun 2018;9:1-16.

53 Smith SM, Nichols TE. Statistical challenges in "big data" human neuroimaging Neuron 2018;97:263-8

54 Bycroft C, Freeman C, Petkova D, et al. The UK Biobank resource with deep phenotyping and genomic data. Nature 2018;562:203-9.

55 LeCun Y, Bengio Y, Hinton G. Deep learning. Nature 2015;521:436-44

56 Weiner MW, Veitch DP, Aisen PS, et al. The Alzheimer's disease neuroimaging initiative 3: continued innovation for clinical trial improvement. Alzheimers Dement 2017:13:561-71.

57 Bearden CE, Thompson PM. Emerging global initiatives in neurogenetics: the enhancing neuroimaging genetics through meta-analysis (ENIGMA) consortium. Neuron 2017;94:232-6

58 Adams NE, Hughes LE, Phillips HN, et al. GABA-ergic dynamics in human frontotemporal networks confirmed by pharmaco-magnetoencephalography. J Neurosci 2020:40:1640-9.

59 Marek K, Jennings D, Lasch S, et al. The Parkinson progression marker initiative (PPMI). Prog Neurobiol 2011;95:629-35.

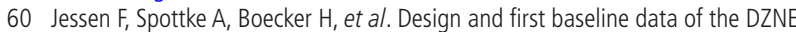
multicenter observational study on predementia Alzheimer's disease (DELCODE). Alzheimers Res Ther 2018:10:1-10. 